\title{
New onset of epileptic seizures induced by Fahr's syndrome secondary to idiopatic hypoparathyroidism.
}

\author{
Cristina Alina Silaghi'1,2, Carmen Emanuela Georgescu², Cristina Ghervan², Ana Valea ${ }^{2}$, Ramona Irimia ${ }^{1}$, Daniel Tudor Cosma ${ }^{3}$, Sanda Petruș \\ Alina Filip ${ }^{5}$, Horațiu Silaghi ${ }^{6}$, Vasile Ţibre ${ }^{7}$ \\ 1 County Emergency Hospital, Cluj-Napoca, Romania; ${ }^{2}$ Departament of Endocrinology "Iuliu Hatieganu" University of Medicine and Pharmacy, Cluj-Napoca, Romania; 3 \\ Diabet, Nutrition and Metabolic Diseases Clinical Center Cluj-Napoca, Romania; ${ }^{4}$ County Hospital, Turda, Romania; ${ }^{5}$ County Hospital, Bistrita, Romania; ${ }^{6} 5^{\text {th }}$ \\ Departament of Surgery, "Iuliu Hatieganu" University of Medicine and Pharmacy, Cluj-Napoca, Romania; ${ }^{7}$ Departament of Neurology, "Iuliu Hatieganu" University of \\ Medicine and Pharmacy, Cluj-Napoca, Romania.
}

\section{Introduction}

Hypocalcemia due to hypoparathyroidism leads to a large spectrum of clinical manifestations but a rare and unusual presentation is onset or aggravation of epilepsy.

Physiological intracranial calcification occurs in about $0.3-1.5 \%$ of cases. It is asymptomatic and detected incidentally by neuroimaging. Pathological basal ganglia calcification is due to various causes, such as: metabolic disorders, infectious and genetic diseases. Hypoparathyroidism and pseudohypoparathyroidism are the most common causes of pathological basal ganglia calcification. We present a representative case study of a spectacular extensive bilateral brain calcifications in the basal ganglia in a patient with idiopathic hypoparatiroidism with neurological manifestations.

\section{Clinical case}

We report a 77-year-old man who was found to have profound hypocalcaemia and idiopatic hypoparathyroidism when investigated for epileptic seizures and loss of consciousness. He had affective disturbances and only mild neurocognitive disorders. Cataract was present. The neurological examination showed an extrapyramidal syndrome with postural tremor and cerebellar ataxia. The deep tendon reflexes were normoactive in all four limbs. Chvostek's sign was present but Trousseau sign was not observed.

Laboratory analysis showed: low concentration of serum ionized calcium at $2.9 \mathrm{mg} / \mathrm{dL}$ (normal: $4.2-5.4 \mathrm{mg} / \mathrm{dL}$ ), total calcium at $5.9 \mathrm{mg} / \mathrm{dL}$ (normal: 8.8 to $10.0 \mathrm{mg} / \mathrm{dL}$ ), hyperphosphoremia at $6.4 \mathrm{mg} / \mathrm{dL}$ (normal: 2.3 to 4.7 $\mathrm{mg} / \mathrm{dL}$ ) and $2.5 \mathrm{pg} / \mathrm{mL}$ intact-parathyroid hormone (normal: 11.0 to 67.0 $\mathrm{pg} / \mathrm{mL}$ ). Alkaline phosphatase, magnesium, calcitonin, serum thyroxin and thyroid-stimulating hormone levels were normal.

Brain computed tomography demonstrated a symmetric, extensive, bilateral calcification of the basal ganglia, centrum semiovale, and bilateral dentate nuclei of the cerebellum, typical for Fahr's syndrome. The red nucleus and substantia nigra appeared normal. The diagnosis of Fahr's syndrome, secondary to hypoparathyroidism was posed. A prepontine meningioma was also found (Figure $1 \mathrm{~A}$ and $\mathrm{B}$ ).

The electrocardiogram showed normal QTc interval and the interictal electroencephalography and electromyography were normal.

Bone densitometry showed increased bone mineral density.

After the patient was treated with oral calcium and active vitamin $D(1$ alphahydroxy vitamin D3), serum calcium levels returned to normal and seizure attacks ceased progressively resulting in stopping antiepileptic drugs.

\section{Conclusions:}

These cases illustrate the importance of search for disrupted phosphocalcic metabolism but also emphasizes the importance of the role of neuro-imaging in patients with new-onset epileptic seizures in order to detect hypocalcemia secondary to hypoparathyroidism.

Bibliography

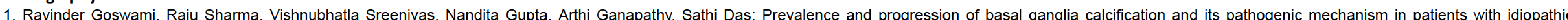
1. Ravinder Goswami, Raju Sharma, Vishnubhatla Sreenivas, Nandita Gupta, Arthi Ganapath
hypoparathyroidism; Clinical Endocrinology; Volume 77, Issue 2 August 2012, Pages 200-206

2. Ramen C. Basak; A Case Report of Basal Ganglia Calcification - A Rare Finding of Hypoparathyroidism; Basak R. OMJ. 24, 220-222 (2009); doi:10.5001/omj.2009.43

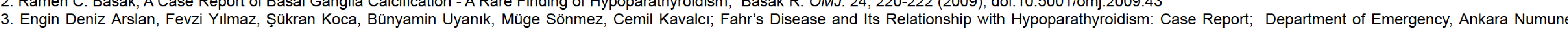
Training and Research Hospital, Ankara, Turkey; JAEMCR 2013; 4: 95-7 doi: 10.5505/jaemcr.2013.59354

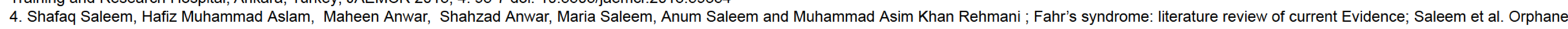
Journal of Rare Diseases 2013, 8:156; http://www.ojrd.com/content/8/1/156 